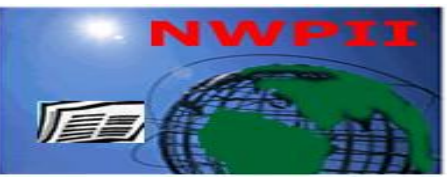

American Journal of Biomedical Sciences

ISSN: 1937-9080

nwpii.com/ajbms

\title{
Assessment of Leucocytes and CD4+ T-cells in HIV Seronegative Tuberculosis Patients
}

\section{Onaiwu T. OHIENGBOMWAN ${ }^{*}$, Rachael OKOJIE ${ }^{2}$, Kelly ELIMIAN ${ }^{2}$, Nosakhare Lawrence IDEMUDIA $^{3}$ and Nosa O. EGHAFONA ${ }^{2}$}

\author{
${ }^{1}$ Redeemer's University Health Services, Osun State, Nigeria \\ ${ }^{2}$ Department of Microbiology, University of Benin, Benin City, Nigeria \\ ${ }^{3}$ University of Benin Teaching Hospital, Benin City, Nigeria \\ "Corresponding Author \\ Onaiwu T. OHIENGBOMWAN \\ Department of Health Services, \\ Redeemer's University, \\ Osun State, \\ Nigeria. \\ Email: royalesteemplc@yahoo.com \\ Phone: +2348039143133
}

Received:13 April 2018;| Revised:11 May 2018; | Accepted: 02 June 2018

\begin{abstract}
Background: Tuberculosis is a major global health problem associated with large mortality. The burden of tuberculosis is particularly high for the African region, mainly due to the high prevalence of Human Immunodeficiency Virus infection. While several studies have focused on the immunological responses of human host to HIV seropositive tuberculosis infection, this study aims to determine the immunological responses (CD4+ and leucocyte cell counts) of human host to HIV seronegative tuberculosis infection. Methods: The study used a cross-sectional study design for population that consisted of 100 study subjects who presented with HIV seronegative tuberculosis infection at diagnosis as well as 40 apparently healthy volunteers who were HIV and tuberculosis negative as control in Central Hospital, Benin City. Results: The result indicated a statistically significant CD4+ lymphocytopaenia, leucocytosis, neutrophilia and monocytosis. Lymphocyte count was not statistically significant despite lymphocytopaenia observed in $28 \%$ of the study subjects. It was observed that 25 (96.2\%) of leucocytosis, 19 (100\%) of neutrophilia, $26(92.9 \%)$ of lymphocytopaenia and $26(92.9 \%)$ of monocytosis were patients having CD4+ lymphocytopaenia. The feminine gender had the highest prevalence rate of CD4+ lymphocytopaenia, leucocytosis, neutrophilia, lymphocytopaenia and monocytosis. Furthermore, disease severity, age and gender seemed to play important role in determining the cellular immunity of tuberculosis patients. Conclusion: CD4+ lymphocytopaenia, leucocytosis, neutrophilia and monocytosis were statistically significant in the study. Interestingly, females appear to be more prone to having CD4+ lymphocytopaenia, leucocytosis, neutrophilia, lymphocytopaenia and monocytosis while these conditions could be occurring in ascending order of age groups.
\end{abstract}




\section{Introduction}

\subsection{Background}

It is a well-known fact that tuberculosis (TB), caused by Mycobacterium tuberculosis (Mtb), has become a serious global health menace which is associated with large number of deaths, usually more than that from any other single infectious disease and has remained one of the top ten causes of death globally ${ }^{[1-2]}$. Mtb is an obligate aerobic, intracellular pathogen, which has a penchant for the lung tissue rich in oxygen supply. The tubercle bacilli gain access into the body via the respiratory route and spread from the site of initial infection in the lungs through the lymphatics or blood to other parts of the body, the apex of the lungs and the regional lymph nodes being preferred sites ${ }^{[3]}$.

An estimated 10.4 million people fell ill with TB in 2016 with about 1.3 million and 374,000 TB deaths occurring among HIV negative and positive people respectively. Africa and South-East Asia accounted for $82 \%$ of the deaths among HIV negative people and $85 \%$ in both HIV negative and positive people ${ }^{[2]}$. WHO report classified Nigeria as one of the 22 high TB-burden countries (HBCs) and the 22 HBCs accounted for over $80 \%$ of the world's TB cases. According to the report, Nigeria has a mortality rate of 94 among HIV negative and 49 among HIV positive cases, prevalence rate of 326 and incidence rate of 338 cases per 100,000

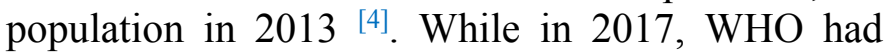
classified Nigeria as one of the 14 high burden countries for combined TB, TB/HIV and MDRTB [2].

It has been reported that most humans who get infected with Mtb do not exhibit progression to the disease and that about one-third of exposed HIV negative individuals become infected, and of this number 3 to $5 \%$ develop TB in the first year, while an additional 3 to $5 \%$ of those infected develop TB later in their lives, it is thought that most adult TB in non-HIV infected patients is caused by reactivation of pre-existing infection ${ }^{[5-6]}$. It is documented that HIV positive persons infected with Mtb have a $50 \%$ chance of developing reactivation (post-primary) TB at some point in their lives, these individuals and others who are immune-suppressed can also be newly infected with Mtb and in many cases show rapid progression to active disease ${ }^{[5-6]}$. Adult TB, whether resulting from activation or new infection in HIV infected patients, is almost always pulmonary and is associated with differing degrees of lung involvement and damage ${ }^{[1]}$.

On the immunological responses to $\mathrm{Mtb}$ invasion, cell-mediated immunity have been implicated to play active role in the body's defense mechanism against Mtb. The cells involved are lymphocytes (CD4+, CD8+ and $\gamma \quad \delta \quad \mathrm{T}$ lymphocytes), neutrophils, monocytes/macrophages, natural killer (NK) cells, dendritic cells and B cells ${ }^{[7-8]}$. Infection with $\mathrm{Mtb}$ is thought to induce distinct antigen-specific CD4+ T-cells endowed with effector functions [9], while monocytes and lymphocytes have been implicated in the induction of immune responses and their levels in peripheral blood is thought to reflect the state of an individual's immunity to infection. Monocytes are an essential component of the innate immune response that acts as a link to the adaptive immune system through antigen presentation to lymphocytes [10]. Studies have demonstrated the functions of neutrophils to include contribution to the generation of effector T-cells, participation in the formation of granuloma, and also play active roles in tissue necrosis, destruction, and infection dissemination ${ }^{[11]}$. Various haematological disorders have been reported in TB to include anaemia, lymphocytopaenia, neutrophilia, monocytosis, monocytopaenia, thrombocytopenia and thrombocytosis ${ }^{[12]}$.

Several studies have focused on the immunological responses of human host to HIV seropositive tuberculosis infection, this present study aims to determine the immunological response of human host to HIV seronegative tuberculosis infection. Specifically, the study was designed to determine the $\mathrm{CD} 4+$ counts, total leucocyte counts (TLC), neutrophil counts (NC), lymphocyte counts (LC) and monocyte counts (MC) in HIV seronegative TB patients attending Central Hospital, Benin City. 


\subsection{Rationale for Study}

TB infections have remained a major public health challenge all over the world because of a large potential reservoir of infection, lack of an effective vaccine to prevent the infection or disease and a long chemotherapeutic regime with multiple chemotherapeutic agents that are difficult to maintain, with significant morbidity and mortality especially with HIV co-infection, multidrug resistant and extensive drug resistant TB infections. There seems to be dearth of data on HIV seronegative tuberculosis infection among victims in Benin City as focus is on HIV co-infected tuberculosis disease. Thus, it is imperative that research focuses on understanding the immune response to this infection. Hence, we were interested in finding out the host immune response to tuberculosis infection in HIV seronegative patients, especially the responses of $\mathrm{CD} 4+$ and leucocytes (total and differential) cell counts.

\subsection{Scope of Study}

This research was restricted to HIV seronegative TB positive (Microscopy and/or GeneXpert) patients at Central Hospital TB Laboratory in Benin City (samples were collected from these patients at the point of diagnosis before the commencement of anti-tubercular therapy-ATT). This was due to time, resources and manpower available for the study. However, the demographic restriction of Central Hospital TB laboratory within Benin City may generate data which may be a true reflection of disease profile in Benin City as well as Edo State and beyond.

\section{Materials and Methods}

\subsection{Ethical considerations}

The Ethics Review Committee of Central Hospital, Benin City reviewed and approved the study protocol (HA.577/Vol.11/76). In addition, information sheet about the study was given or explained to each study participant to have a full understanding of the research as well as what is expected of them. In the scenario where a potential study participant was not literate, the information sheet was explained in simple English, Pidgin English or local dialect (Bini). Thereafter, consent (written or verbal) was sought from study participants.

\subsection{Study location and Population}

The research study was conducted among patients attending Central Hospital, Benin City, Edo State, Nigeria. The study population consisted of one hundred and forty subjects (140 participants).

\subsection{Sample collection and processing}

Six milliliter $(6 \mathrm{ml})$ of blood sample was aseptically drawn from each of the participants by veni-puncture; $4 \mathrm{ml}$ of the blood sample was dispensed into Ethylene diamine tetra-acetic acid (EDTA) anticoagulated container and mixed properly to avoid clotting and the remaining $2 \mathrm{ml}$ was dispensed into plain container and allowed to clot. Serum was separated from the clotted blood sample in the plain container and stored at $4^{\circ} \mathrm{C}$ which was then used for HIV screening. The stored serum sample prior to use was allowed to attain room temperature.

CD4+ count: CD4+ count was determined by the Partec flow-cytometric method using an EDTA whole blood sample. The instrument uses fluorescent-labelled anti-CD4 monoclonal antibodies to capture CD4+ T-cells from whole blood and allows automated CD4+ counting.

Twenty Microliter $(20 \mu 1)$ of CD4+ monoclonal antibody was introduced into Rohren test tubes and $20 \mu 1$ of well mixed whole blood of study subjects was added, this was mixed and incubated in the dark for 15 minutes at room temperature. Thereafter, $800 \mu 1$ of CD4+ buffer was added, mixed and read on the Cyflow. Partec ${ }^{\mathrm{TM}}$ Flow Cytometers (CyFlow ${ }^{\circledR}$ and PAS $($ ) is a compact flow cytometer for diverse flow cytometry analysis whereby cells in suspension is differentiated and counted according to the cell size and internal structure.

Total/differential leucocyte count: Full blood count (complete blood count) was determined in EDTA blood containers using Sysmex kx-21N haematology auto-analyzer instrument. The equipment was switched on and set to the whole blood mode, sample information was appropriately imputed into the machine while the blood sample was mixed sufficiently in the EDTA container. The tube was then set to the sample probe and in that condition the start switch was pressed and the 
system executes automatic analysis and displays the result on the LCD screen. A printed copy of the result was obtained at the end of the analysis from which total and differential leucocyte cell counts were extracted from the parameters.

The instrument analyzes whole blood samples by flow cytometry using an argon laser as the light source, whole blood samples are stained with a fluorescent dye and passed through laser beam in the sheathed flow cell. The fluorescently labeled cells were irradiated using an argon laser beam, the forward scatter and the side fluorescence emitted from each cell in the sheath flow were detected while the former (forward scatter) was used as an indicator of relative cell size, the later (side fluorescence) was used as an indicator of RNA content. All red blood cells in the sample were haemolyzed while the white blood cells were denucleated and their nuclei shrunk for differential enumeration.

\subsection{HIV Screening}

The 140 subjects were screened for HIV using Alere Determine TM HIV-1/2 to detect HIV seronegative cases. The reagents and samples were removed from the refrigerator and allowed to stand for about 25minutes in order to attain room temperature. Test device numbering 140 were labelled appropriately, a precision pipette was used to apply $50 \mu 1$ of the serum to the sample pad and allowed to migrate by capillary action through the conjugate pad and then through the nitrocellulose membrane. A timer was set and the results were read in 25 minutes to determine HIV positive and negative cases. HIV negative cases were included in the study while the positive cases were excluded.

\subsection{Selection criteria}

Subjects used in this study included TB positive (microscopy and/or GeneXpert) patients without HIV infection. For the purpose of this study, the following groups of people were excluded:

1. HIV seropositive TB patients.

2. Children who are below the ages of 15 years of age due to their inability to pass sputum for TB test.

3.Pregnant female patients to avoid interference of pregnancy with leucocyte count.

\subsection{Statistical analysis}

The data generated from the laboratory analysis of blood samples collected from the study and control subjects were grouped according to the value presentation, that is, normal values and low/high values. In order to test the hypotheses stated for the study, the student's unpaired t-test (Microsoft Excel Package, 2013 version) was used for analyses. The P-value was taken as $0.05,95 \%$ confidence interval, such that $\mathrm{P}<0.05$ indicated a significance.

\section{Results}

As shown in Table 1, 43\% of the study subjects presented with CD4+ lymphocytopaenia, while $26 \%, 19 \%, 28 \%$ and another $28 \%$ presented with leucocytosis, neutrophilia, lymphocytopaenia and monocytosis respectively.

Table 1: Result/Statistical Analysis of $\mathrm{CD}_{4}{ }^{+}$and Leucocyte counts of study subjects

\begin{tabular}{llclc}
\hline Parameter & NV (\%) & Ab (\%) & P-value \\
\hline CD $^{+}$ & 57 & 43 (CD4+ lymphocytopaenia) & 0.000 \\
TLC & 74 & 26 (Leucocytosis) & 0.002 \\
NC & 81 & 19 (Neutrophilia) & 0.049 \\
LC & 72 & 28 (Lymphocytopaenia) & 0.658 \\
MC & 72 & 28 (Monocytosis) & 0.003
\end{tabular}

KEY: TLC- Total leucocyte count; NC- Neutrophil count; LC- Lymphocyte count; MC- Monocyte count; NVNormal values; Ab- Abnormal values. 
Table 2 showed that $43 \%$ of the study subjects had CD4+ counts < 500cells/ $\mu 1$ (min-max: 378-496 cells $/ \mu 1), \quad 51 \%$ had between 500 cells $/ \mu 1$ and 999 cells $/ \mu 1$ while $6 \%$ had greater than 1000 cells $/ \mu 1$ (min-max: 1076-1301 cells/ $\mu 1$ ). It is also seen that $25(96.2 \%)$ of the abnormal values of TLC, $19(100 \%)$ of the abnormal values of NC, $26(92.9 \%)$ of the abnormal values of LC and $26(92.9 \%)$ of the abnormal values of $\mathrm{MC}$ occurred within the abnormal values of CD4+ count of the study subjects while only $1(3.8 \%)$ of the abnormal TLC value, $2(7.1 \%)$ of the abnormal $\mathrm{LC}$ value and $2(7.1 \%)$ of the abnormal MC value occurred within the normal values of CD4+ count.

Table 2: Summary of $\mathrm{CD}_{4}^{+}$counts and distribution of abnormal leucocyte counts of study subjects

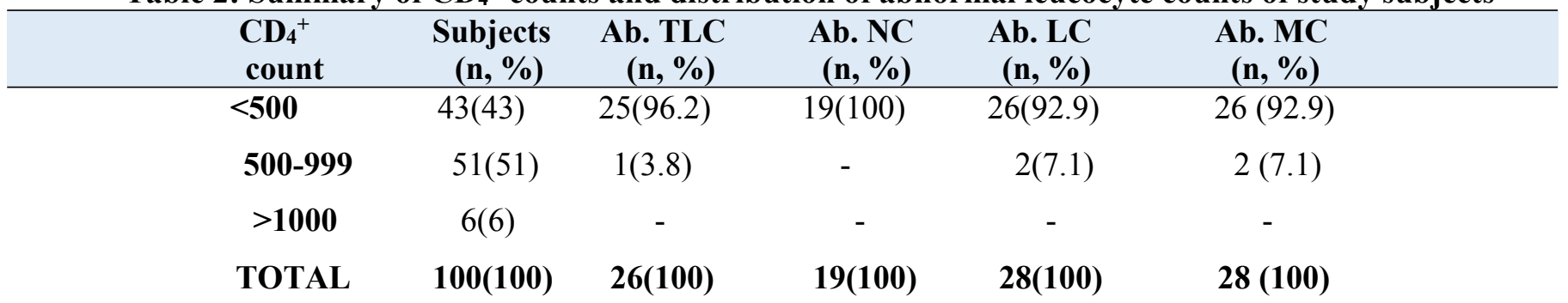

KEY: TLC- Total leucocyte count; NC- Neutrophil count; LC- Lymphocyte count; MC- Monocyte count; AbAbnormal values; $n$ - Number

As shown in Table 3, CD4+ lymphocytopaenia was observed in $32(52.5 \%)$ females and $15(38.5 \%)$ males while 29(47.5\%) females and 24(61.5\%) males had normal CD4+ count.

Table 3: Gender distribution of $\mathrm{CD}_{4}{ }^{+}$counts of study subjects

\begin{tabular}{llll}
\hline CD $_{\mathbf{4}}^{+}$Count & F (n, \%) & M (n, \%) & TOTAL \\
\hline$<\mathbf{5 0 0}$ & $32(52.5)$ & $15(38.5)$ & 43 \\
$\mathbf{5 0 0 - 9 9 9}$ & $26(42.6)$ & $21(53.8)$ & 51 \\
$>\mathbf{1 0 0 0}$ & $03(4.9)$ & $03(7.7)$ & 06 \\
TOTAL (\%) & $\mathbf{6 1 ( 1 0 0 )}$ & $\mathbf{3 9 ( 1 0 0 )}$ & $\mathbf{1 0 0}$
\end{tabular}

KEY: F- Female; M- Male, n- Number

Table 4 showed that $29.5 \%$ of females and $20.5 \%$ of males had leucocytosis, $21.3 \%$ of females and $15.4 \%$ of males had neutrophilia, $29.5 \%$ of females and $25.6 \%$ of males had lymphocytopaenia, and $32.8 \%$ of females and $20.5 \%$ of males had monocytosis.

Table 4: Gender distribution of leucocyte counts of study subjects

\begin{tabular}{lllllll}
\hline \multirow{2}{*}{ Parameter } & \multicolumn{3}{c}{ Abnormal values } & \multicolumn{3}{c}{ Normal values } \\
\cline { 2 - 7 } & No & F (n, \%) & M (n, \%) & No & F (n, \%) & M (n, \%) \\
\hline TLC & 26 & $18(29.5)$ & $8(20.5)$ & 74 & $43(70.5)$ & $31(79.5)$ \\
NC & 19 & $13(21.3)$ & $6(15.4)$ & 81 & $48(78.7)$ & $33(84.6)$ \\
LC & 28 & $18(29.5)$ & $10(25.6)$ & 72 & $43(70.5)$ & $29(74.4)$ \\
MC & 28 & $20(32.8)$ & $8(20.5)$ & 72 & $41(67.2)$ & $31(79.5)$ \\
\hline
\end{tabular}


In relation to age, it was observed from table 5 that age group 51-60 years had highest CD4+ lymphocytopaenia (54.5\%), followed by 41-50years (48.3\%), 31-40years (46.2\%), 21-30years (25\%) and age group <20years (20\%). For TLC distribution in relation to age group, it was observed that 51-60years had the highest leucocytosis (36.4\%), followed by 31-40years $(28.2 \%)$, 4150years $(27.6 \%)$ and 21-30years (18.8\%). Age group 31-40years had the highest record of neutrophilia (25.6\%), followed by 51-60years (18.2\%), 41-50years (17.2\%) and 21-30years (12.5\%). Age group 51-60years had the highest record of lymphocytopaenia $(36.4 \%)$, followed by $31-40$ years $(33.3 \%), 41-50$ years $(27.6 \%)$ and $21-$ 30years (18.8\%). Age group 51-60years had the highest record of monocytosis $(36.4 \%)$, followed by 31-40years $(33.3 \%), 41-50$ years $(27.6 \%)$ and 21 30years (18.8\%). Age group less than 20years had no record of abnormal leucocyte values.

Table 5: Normal and abnormal values among age groups of study subjects

\begin{tabular}{|c|c|c|c|c|c|}
\hline Age & $\begin{array}{c}<20 \\
(n, \%)\end{array}$ & $\begin{array}{r}21-30 \\
(n, \%)\end{array}$ & $\begin{array}{l}31-40 \\
(n, \%)\end{array}$ & $\begin{array}{l}41-50 \\
(n, \%)\end{array}$ & $\begin{array}{r}51-60 \\
(n, \%)\end{array}$ \\
\hline \multicolumn{6}{|c|}{ Abnormal values } \\
\hline CD4+ & $1(20)$ & $4(25)$ & $18(46.2)$ & $14(48.3)$ & $6(54.5)$ \\
\hline TLC & 0 & $3(18.8)$ & $11(28.2)$ & $8(27.6)$ & $4(36.4)$ \\
\hline $\mathrm{NC}$ & 0 & $2(12.5)$ & $10(25.6)$ & $5(17.2)$ & $2(18.2)$ \\
\hline $\mathbf{L C}$ & 0 & $3(18.8)$ & $13(33.3)$ & $8(27.6)$ & $4(36.4)$ \\
\hline MC & 0 & $3(18.8)$ & $13(33.3)$ & $8(27.6)$ & $4(36.4)$ \\
\hline \multicolumn{6}{|c|}{ Normal values } \\
\hline CD4+ & $4(80)$ & $12(75)$ & $21(53.8)$ & $15(51.7)$ & $5(45.5)$ \\
\hline TLC & $5(100)$ & $13(81.2)$ & $28(71.8)$ & $21(72.4)$ & $7(63.6)$ \\
\hline NC & $5(100)$ & $14(87.5)$ & $29(74.4)$ & $24(82.8)$ & $9(81.8)$ \\
\hline $\mathbf{L C}$ & $5(100)$ & $13(81.2)$ & $26(66.7)$ & $21(72.4)$ & $7(63.6)$ \\
\hline MC & $5(100)$ & $13(81.2)$ & $26(66.7)$ & $21(72.4)$ & $7(63.6)$ \\
\hline TOTAL & 5 & 16 & 39 & 29 & 11 \\
\hline
\end{tabular}

KEY: TLC- Total leucocyte count; NC- Neutrophil count; LC- Lymphocyte count;

MC- Monocyte count; n- Number

\section{Discussion}

$\mathrm{TB}$ is a major health problem throughout the world and remains the single largest infectious disease causing high mortality in humans ${ }^{[1-2]}$. The immune response to this infection relies on cellmediated immunity which is evident in CD4+ and CD8+ T-lymphocyte responses to Mtb antigens ${ }^{[7-8]}$.

This study was to enumerate $\mathrm{CD} 4+$ lymphocytes and leucocytes (total and differential) in HIV seronegative TB positive (Microscopy and/or GeneXpert) patients before the commencement of anti-tubercular therapy (ATT). CD4+ count was found to be significantly reduced (CD4+ lymphocytopaenia) below the 500cells $/ \mu \mathrm{L}$ threshold mark in $43 \%$ of the study subjects while $57 \%$ had CD $4+$ counts above 500 cells $/ \mu \mathrm{L}$. The statistical analysis of the CD4+ counts against control subjects showed a statistically significant $(\mathrm{P}<0.05) \mathrm{CD} 4+$ lymphocytopaenia.

The findings from this study are in agreement with the findings of other researchers ${ }^{[13-18]}$. A similar study conducted in Ethiopia ${ }^{[16]}$ reported a substantial prevalence of low CD4+ cell counts 
before the initiation of ATT with $25 \%$ having CD4+ cell counts below 500cells $/ \mu \mathrm{L}$ and $10 \%$ had CD4+ cell counts lower than 350 cells $/ \mu \mathrm{L}$. The present study did not have any record of CD4+ cells below 350 cells $/ \mu \mathrm{L}$, this may probably be due to the severity of disease between the two studies as the present study focused on patients with less severe tuberculosis infection as none of the patients were on clinical admission and not on ATT at the time of the study so as to prevent the interaction of drugs (ATT) with the outcome of the study. The researchers correlated CD4+ cell strata with clinical variables and concluded that low CD4+ cell counts are associated with TB disease severity, a situation that cannot be determined in this present study due to the fact that all the study subjects were those coming for TB diagnostic test for the first time and severity of disease was not the focus of the study.

In another study in Iran [14] and Saudi Arabia [15], the researchers reported significantly $(\mathrm{P}<0.05)$ lower CD4+ counts among TB infected HIV negative patients as compared with control. Another study carried out in Ethiopia ${ }^{[17]}$ and similar studies conducted in Nigeria [18-19], supported the CD4+ lymphocytopaenia findings from this present study.

Sixty one (61) females and 39 males were used for the study, $32(52.5 \%)$ females and 15(38.5\%) males had CD4+ lymphocytopaenia (table 3). It can be observed from the findings of this study with regards to gender that more females were prone to CD4+ lymphocytopaenia compared to males. A similar study ${ }^{[18]}$ reported a significant higher mean of CD4+ cells in female TB patients on DOTS than their male counterparts but reported no significant difference in gender of TB patients not on ATT. The CD4+ lymphocytopaenia observed in this study from patients suffering from tuberculosis can be a sign of CD4+ T-cell suppression.

The haematological profile of HIV seronegative tuberculosis patients carried out in this study before the commencement of ATT showed a statistically significant $(\mathrm{P}<0.05)$ leucocytosis when compared with those of control subjects. The results showed 26\% leucocytosis among study subjects, there was however no record of leucopaenia in this study. The findings of this study support the findings of other researchers who also reported significant leucocytosis $(\mathrm{P}<0.05)$ in their study $[18$, 20-21]. However, the statistically significant leucocytosis reported in this study is at variance with the findings of other researchers who found normal leucocyte values in their study [22-23], the disparity in the findings could be due to normalization of leucocytes as a result of therapy as the researchers study subjects included those already on ATT, while other researchers like Shafee and colleagues ${ }^{[24]}$ reported $14 \%$ leucopaenia in their study. The leucocytosis observed in our study is probably due to the increased response of leucocytes as a result of its phagocytic activity to Mtb infection.

The neutrophils counts of HIV seronegative tuberculosis patients observed in this study showed a statistically significant $(\mathrm{P}<0.05)$ neutrophilia when compared with those of control subjects. The results showed that $19 \%$ of the study subjects had neutrophilia, there was no neutropaenia recorded in this study. The findings of this study supports the findings of other researchers ${ }^{[18,20-21]}$ and contrary to the normal values reported in another study ${ }^{[23]}$.

The lymphocyte counts of HIV seronegative tuberculosis patients carried out in this study before the commencement of ATT was not statistically significant $(\mathrm{P}>0.05)$ despite the lymphocytopaenia recorded in $28 \%$ of the study subjects. This is in support of the findings of other researchers [18, 20-21, 23]. However, there was no record of lymphocytosis in this study contrary to the $6 \%$ lymphocytosis reported in one study ${ }^{[25]}$.

The monocyte counts of HIV seronegative tuberculosis patients carried out in this study before the commencement of ATT showed a statistically significant $(\mathrm{P}<0.05)$ monocytosis, the results showed that $28 \%$ of the study subjects had monocytosis, there was however no monocytopaenia recorded in this study. The findings of this study agrees with previous studies [20-21], but contrary to the no significant difference between study and control groups reported in another study ${ }^{[23]}$.

It can be seen that the leucocytosis observed in this study is due to neutrophilia and monocytosis, the roles of neutrophils and monocytes in tuberculosis infection cannot be undermined as they are soon mobilized to the site of infection to provide cellular immune cover for the host. Another observation from our study (table 2) is that CD4+ lymphocytopaenia in HIV seronegative tuberculosis 
subjects affects the total and differential leucocyte counts of sufferers as study subjects with normal CD4+ cells had relatively stable total and differential leucocyte counts compared to study subjects with CD4+ lymphocytopaenia.

CD4+ lymphocytopaenia was found to be variedly distributed among different age. While, age group 51-60years had the highest prevalence rate $(54.5 \%)$ age group <20years had the lowest prevalence rate $(20 \%)$. An increasing prevalence rate of $\mathrm{CD} 4+$ lymphocytopaenia was observed in ascending order of age groups, this is suggestive of diminishing cellular immunity with increase in age from age group <20years to 51-60years. This findings corroborates the findings of other researchers ${ }^{[26-27]}$. Age group 51-60years was found to have the highest prevalence rate for leucocytosis, lymphocytopaenia and monocytosis while age group 31-40years had the highest prevalence rate for neutrophilia. This observation is suggestive of reduced cellular immunity with regards to increase in age.

\section{Conclusion}

This study highlights the importance of cellular immunity conducted by CD4+ T-cells and leucocytes in the outcome of tuberculosis. The findings from this study indicated a statistically significant CD4+ lymphocytopaenia, leucocytosis, neutrophilia, monocytosis and a non-statistically significant lymphocytopaenia despite $28 \%$ lymphocytopaenia recorded in the study. The feminine gender had the highest prevalence rate of CD4+ lymphocytopaenia, leucocytosis, neutrophilia, lymphocytopaenia and monocytosis. An increasing prevalence rate of $\mathrm{CD} 4+$ lymphocytopaenia was observed in ascending order of age groups.

\section{Recommendation}

The response of cellular immunity should always be considered in approach to patients with tuberculosis and the need for modalities such as effective vaccination and immunotherapy should be implemented while CD4+ and leucocyte values should always be monitored during the course of ATT.

\section{Acknowledgement}

The authors wish to appreciate the cooperation of Central Hospital TB Laboratory staff and the University of Benin Teaching Hospital ART Laboratory.

\section{References}

1 Mohajan, H.K. Tuberculosis is a Fatal Disease among Some Developing Countries of the World. American Journal of Infectious Diseases and Microbiology, 2015, 3(1), 18-31.

2 World Health Organization. Global tuberculosis report-executive summary; WHO Press: Geneva, Switzerland, 2017; pp 1-4.

3 Raja, A. Immunology of tuberculosis. Indian Journal of Medical Research, 2004, 120, 213232.

4 World Health Organization . Global Tuberculosis Report, WHO Press: Geneva, Switzerland, 2014; pp 1-171.

5 Ahmad S. Pathogenesis, immunology, and diagnosis of latent Mycobacterium tuberculosis infection. Clin Dev Immunol 2011; 2011: 814943 [PMID: 21234341 PMCID: PMC3017943 DOI: $10.1155 / 2011 / 814943]$

6 Sontakke, P.C.; Waghmode, J.P.; Khade, A.A. A Brief Review on Pathophysiology and Treatment of HIV and TB Co-Infection, International Journal of Science and Research Methodology, 2015, 1(1), 49-59.

7 Zuniga J, Torres-Garcia D, Santos-Mendoza T, Rodriguez-Reyna TS, Granados J, Yunis EJ. Cellular and humoral mechanisms involved in the control of tuberculosis. Clin Dev Immunol 2012; 2012: 193923 [PMID: 22666281 PMCID: $\quad$ PMC3362816 DOI: 10.1155/2012/193923]

8 Lerner TR, Borel S, Gutierrez MG. The innate immune response in human tuberculosis. Cell Microbiol 2015; 17(9): 1277-1285 [PMID: 26135005 PMCID: PMC4832344 DOI: $\underline{10.1111 / \mathrm{cmi} .12480]}$

9 Mpande CAM, Dintwe OB , Musvosvi M, Mabwe S, Bilek N, Hatherill M, Nemes E, Scriba TJ, Team SCI. Functional, AntigenSpecific Stem Cell Memory (TSCM) CD4(+) $\mathrm{T}$ Cells Are Induced by Human 
Mycobacterium tuberculosis Infection. Front Immunol 2018; 9: 324 [PMID: 29545791 PMCID: $\quad$ PMC5839236 DOI: 10.3389/fimmu.2018.00324]

10 Wang J, Yin Y, Wang X, Pei H, Kuai S, Gu L, Xing H, Zhang Y, Huang Q, Guan B. Ratio of monocytes to lymphocytes in peripheral blood in patients diagnosed with active tuberculosis. Braz J Infect Dis 2015; 19(2): 125-131 [PMID: 25529365 DOI: 10.1016/j.bjid.2014.10.008]

11 Lyadova IV. Neutrophils in Tuberculosis: Heterogeneity Shapes the Way? Mediators Inflamm 2017; 2017: 8619307 [PMID: 28626346 PMCID: PMC5463159 DOI: 10.1155/2017/8619307]

12 Iqbal , S.; Ahmed, U.; Khan, M.A. Haematological Parameters altered In Tuberculosis, Pakistan Journal of Physiology, 11(1), 13-16.

13 Uppal SS, Tewari SC, Verma S, Dhot PS. Comparison of CD4 and CD8 lymphocyte counts in HIV-negative pulmonary TB patients with those in normal blood donors and the effect of antitubercular treatment: hospitalbased flow cytometric study. Cytometry B Clin Cytom 2004; 61(1): 20-26 [PMID: 15351978 DOI: 10.1002/cyto.b.20018]

14 Davoudi S, Rasoolinegad M, Younesian M, Hajiabdolbaghi M, Soudbakhsh A, Jafari S, EmadiKouchak H, Mehrpouya M, Lotfi $\mathrm{H}$. CD4+ cell counts in patients with different clinical manifestations of tuberculosis. Braz $\boldsymbol{J}$ Infect Dis 2008; 12(6): 483-486 [PMID: 19287835]

15 Al-Aska, A.I.; Al-Anazi, A.R.; Al-Subaei,S.S.; Al-Hedaithy, M.A.; Barry, M.A.; Somily, A.M.; Buba, F.; Yusuf, U.; Al-Anazi, N.A. CD4+ T-Lymphocytopenia in HIV Negative Tuberculous Patients at King khalid University Hospital in Riyadh, Saudi Arabia, European Journal of Medical Research, 2011, 16, 285288.

16 Skogmar S, Schon T, Balcha TT, Jemal ZH, Tibesso G, Bjork J, Bjorkman P. CD4 cell levels during treatment for tuberculosis (TB) in Ethiopian adults and clinical markers associated with CD4 lymphocytopenia. PLoS One 2013; 8(12): e83270 [PMID: 24358268
PMCID: $\quad$ PMC3865172

DOI:

10.1371/journal.pone.0083270]

17 Atomsa D , Abebe G, Sewunet T. Immunological markers and hematological parameters among newly diagnosed tuberculosis patients at Jimma University Specialized Hospital. Ethiop J Health Sci 2014; 24(4): 311-318 [PMID: 25489195 PMCID: PMC4248030]

18 Ositadimma, I.M.; Samuel, C.M.; Bright, O.E.; Okechukwu, O.C. Some Haematological Parameters and Immunoglobulin Levels in Mycobacterium tuberculosis Infected Patients in Benin City, Southern Nigeria, International Journal of Tropical Disease and Health, 2017, 26(1), 1-9.

19 Amilo, G.I.; Meludu, S.C.; Onyenekwe, C.; Ele, P.U.; Ezechukwu, C.; Ekejindu, G.O.C.; Ifeanyichukwu, M.O.; Nwobodo, E. Evaluation of CD4+ and CD8+ T lymphocyte counts and serum levels of immunoglobulins in pulmonary tuberculosis (PTB) patients with or without HIV coinfection in South Eastern Nigeria, African Journal of Pharmacy and Pharmacology, 2012, 6(23), 1639-1643.

20 Al-Omar, I.A.; Al-Ashban, R.M.; Shah, A.H. Haematological abnormalities of Saudis suffering from pulmonary tuberculosis and their response to treatment, Research Journal of Pharmacology 2009, 3(4), 78-85.

21 Amilo, G.I.; Meludu, S.C.; Ele, P.U.; Ezechukwu, C.; Onyenekwe, C.; Ifeanyichukwu, M. Haematologic Indices in Pulmonary Tuberculosis with or without HIV Co-infection in South Eastern Nigeria, Advances in Life Science and Technology, 2013, 11, 1-7.

22 Akpan, P.A.; Akpotuzor, J.O.; Akwiwu, E.C. Some Haematological Parameters of Tuberculosis (TB) Infected Africans: The Nigerian Perspective, Journal of Natural Sciences Research, 2012, 2(1), 50-56.

23 Hager, A.S.; Nizar, R.M.A. Abnormalities of hematological parameters in newly diagnosed pulmonary tuberculosis patients in Kirkuk city, Journal of Babylon University/Pure and Applied Sciences, 2012, 5(20), 1486-1492.

24 Shafee M, Abbas F, Ashraf M, Alam Mengal M, Kakar N, Ahmad Z, Ali F. Hematological 
profile and risk factors associated with pulmonary tuberculosis patients in Quetta, Pakistan. Pak J Med Sci 2014; 30(1): 36-40 [PMID: 24639827 PMCID: PMC3955538 DOI: 10.12669/pjms.301.4129]

25 Akintunde EO, Shokunbi WA, Adekunle CO. Leucocyte count, platelet count and erythrocyte sedimentation rate in pulmonary tuberculosis. Afr J Med Med Sci 1995; 24(2): 131-134 [PMID: 8669390]
26 Oladepo DK, Idigbe EO, Audu RA, Inyang US, Imade GE, Philip AO, Okafor GO, Olaleye D, Mohammed SB, Odunukwe NN, Harry TO, Edyong-Ekpa M, Idoko J, Musa AZ, Adedeji A, Nasidi A, Ya'aba Y, Ibrahim K. Establishment of reference values of CD4 and CD8 lymphocyte subsets in healthy Nigerian adults. Clin Vaccine Immunol 2009; 16(9): 1374-1377 [PMID: 19641097 PMCID: PMC2745013 DOI: 10.1128/CVI.00378-08].

27 Dash, M. ; Padhi, S. ; Panda, P.; Parida, B .; Patra, G.C. Immunophenotypic enumeration of CD4+ T-lymphocyte values in human immunodeficiency virus-seronegative adults in Eastern India, International Journal of Medicine and Biomedical Research, 2012, 1(3), 242-249. 